\title{
The Manifestation of National Collective Unconsciousness and Expectation Vision in Non-Legacy Products -------Take the Nanan Yingdu Pull-Out Lamp in Fujian as an Example
}

\author{
Lin Zibo ${ }^{1, a}$, Yang Peiyu ${ }^{2, b^{*}}$ \\ ${ }^{1}$ Xiamen Academy of Arts and Design, Fuzhou University, Xiamen, Fujian, China \\ ${ }^{2}$ Xiamen Academy of Arts and Design, Fuzhou University, Xiamen, Fujian, China \\ a57762087@qq.com \\ ${ }^{b * 4} 489071181 @ q q . c o m$
}

\begin{abstract}
Cultural and creative products such as non-legacy are the accumulation of national culture. In the design process, the culture of 5,000 years in China is hidden in a collective unconscious form. The color, shape, texture, and text content of the work awaken people's hearts. Primitive images, these all affect people's purchasing behavior; at the same time, can contemporary non-legacy cultural creations properly meet people's expectations, and innovate people's original primitive images without "lonely voice", so that the collective unconscious can get New cultural carrier. This article takes the Nanan Yingdu lamp pulling folk event in Fujian Non-Heritage Project as an example to investigate the reflection of the image of auspicious and festive lanterns in traditional Chinese traditional culture in the collective unconsciousness of contemporary people, and comprehensively discusses the In the era, how Chinese culture retains its non-legacy cultural characteristics in the torrent of culture and is not obliterated by foreign cultures is a question worth considering for contemporary art workers.
\end{abstract}

Keywords: Non-legacy products, collective unconsciousness, expectation horizon

\section{非遗文创产品中民族集体无意识与期待视野的彰显 一以福建南安英都拔拔灯为例}

林梓波 ${ }^{1,}$, 杨沛余 ${ }^{2, \text { b* }}$

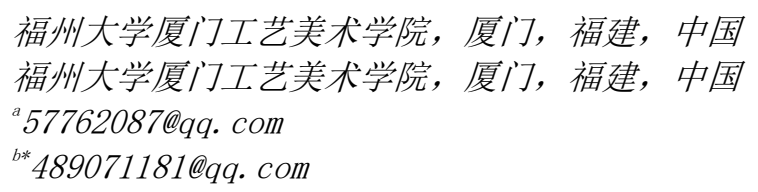

\section{摘要:}

非遗等文创产品是民族文化的积淀，在其设计过程中中华上下五千年的文化 以一种集体无意识的形态潜 藏在其中，作品的色彩、造型、纹理、文字内容都唤醒人们心中的原始意象，这些都影响着人们的购买 行为; 同时当代非遗文创能否 恰如其分的满足人们的期待视野，在不是曲高和寡的情况下创新人们原有 的原始意象，使集体无意识在当代社会中得到新的文化载体。文章以福建非遗项目中的南安的英都拔拔 灯民俗活动为例, 调查传统中国传统文化中象征吉祥喜庆的灯笼的意象在当代人们的集体无意识中的反 映, 并综合论述在多元文化的时代中，中华文化如何在文化的洪流中，保留自己的非遗文化特色，不被 外来文化所湮没是当下文艺工作者值得思考的问题。

关键词: 非遗文创产品; 集体无意识; 期待视野 


\section{1. 前言}

非遗作为中国劳动人民创造性实践的产物, 丰富了我 国绚丽多彩的艺术文化 宝库, 并且将强大的中国文化带 到海外，彰显了强大的中国文化实力，当代中国 非遗在 继承和弘扬中国文化方面有着不容忽视的地位; 其次, 非 遗当中许多工艺 美术品作为国内外贸易的产品, 加速了 资本的周转, 成为中国经济发展中非常重 要的一股力量, 对发展和巩固了中国的制造产业有着积淀的作用。如今随 着现代科学技术不断发展, 非遗作为民族文化的体现彰显 了千百年来民族集体无意识的传承。福建南安英都镇的拔 拔灯自明代万历年间滥解, 在已经传承了 500 多年的历 史, 英都最早作为中国古代 “海上丝绸之路的” 的内河驿 站, 由于河道 “九曲十八弯” 水流湍急, 来往的航船需要 靠岸停泊, 逆水行舟时的时候就需要船夫进行拉纤, 后来 这种劳动实践与游灯闹新春的民俗活动融合到一起, 祈祷 河运顺利。如今每年的正月初九, 来自不同村舍的队伍使 用长绳作为纽带将灯笼贯穿起来, 最前面的人弯着腰肩负 绳索缓慢前行, 仿佛生动的还原了中国古代纤夫拉船的画 面, 同时在拔拔灯活动期间, 也会伴随着拍胸舞、大鼓队 等闽南民俗表演活动, 英都 “拔拔灯” 这一活动不仅成为 了人们群众喜迎新春的重要活动, 而且成为了乡村文化中 重要的因素, 成为了思乡一种羁绊, 并被列为了第二批国 家级非物质文化遗产。

\section{2. 非遗的历史回响}

在当代信息社会, 各种文化通过不同的载体进入中 国, 如好莱坞电影、说唱音乐等大众媒体, 这种外来文化 以无意识的方式不断侵蚀着民族文化, 法国最先 意识到 这种文化侵略并且开始限制外来影片, 来保证自己本国文 化的发展。中国 作为非遗的大国, 拥有着悠久的历史文 化, 同时各种传统工艺在吸收科技成果下 成为新的文化 载体, 如中国台北故宫的 “朕知道了” 的一款胶带采用了 红、黄、白三种颜色, 其中红色使人回想到朱柱碧瓦、 黄色作为古代中国皇室象征使人自然而 然想到皇帝、白 色放佛皇上在宣纸上进行了书写; 北京故宫倡导的是一种 紫禁城 生活美学, 其中北宋王希孟的《千里江山图》被 制作成了手提袋、桌垫、装饰画、茶具套装等, 这不仅 彰显了中国文化和中国优秀绘画, 作品而且使人们对生活 方 式有了更高的要求。中国各地也相继推出各种的文创 产品, 特别是各地的古乡古 镇, 如乌镇、周庄、凤凰等 也利用当地具有特色的服饰、工艺品、乐器来进行非遗文 创产品设计。通过非遗文创产品能了解各地的习俗、文化 等, 就彰显了民族 的集体无意识, 唤醒人们对原始意象 的回响。

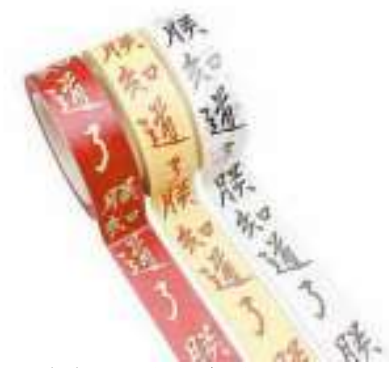

图 1 图片来自于网络

\section{3. 荣格的集体无意识与姚斯的期待视野}

\section{1. 集体无意识}

荣格的分析心理学理论与姚斯的期待视野可以帮助 我们分析传统文化的原始意象与当代文创产品创新。荣格 的将人格分为意识、个人无意识和集体 无意识三层, 并 且荣格在其 1922 年《论分析心理学与诗的关系》中提出, 人的无意识有个体的和非个体（或超个体）的两个层 面........ 后者则包括婴儿实际开始以前的全部时间, 即包括祖先生命的残留, 它的内容能在一切同类人的心中 找到, 带有普遍性, 故称集体无意识 ${ }^{[1]}$ 。荣格为集体无意 识所下的定义是：“集体无意识是人类心理的一部 分.......而集体无意识主要由 “原型” 所组成。” ${ }^{22}$

荣格向人们说明原型与原始意象并不相同, 原型是指 一种与生俱来的心理模 式, 所有的原型的集合构成了集 体无意识, 而原始意象介于原型与意象等感性材 料之间, 可以规范和限定意象因此两者构成一种潜在与外显的关 系。 ${ }^{[3]}$ 正是在这种 相互关系下, 人们可以通过原始意象 一例如云龙风鹤的纹样, 可以使人联想到 吉祥如意、 帝王富贵的织物一一激发起深藏在人们脑海中的集体无 意识, 这种集体无意识正是人们在中国传统文化的熏陶下 逐渐培养起来的。当代设计产品层出不穷, 我们可以利用 原始意象等感性符号, 来设计具有中国文化特色的产品, 使中国文化在新时代焕发光芒。

\section{2. 期待视野}

姚斯作为德国接受美学的代表与奠基人,他所提倡的 期待视野, 将艺术鉴赏的重心由作品、作者转向了读者、 鉴赏者。姚斯曾经指明: “一部文学作品....视野的变 化'之间的距离, 决定着文学作品的艺术特性。 ${ }^{[4]}$ ” 其中 这里视野变化之间的举例可以分为两个方面来讲, 其一, 是指读者的期待视野与新作品之间, 应当有一个适度的审 美距离, 一方面作品应 当是读者出乎意料之外, 超出他 原本的期待视野, 是读者对其感到新奇, 因为心 的体验 扩大了其审美想象空间、创新了长期的审美经验, 这正是 艺术设计作品以 创新为核心的关键所在; 另一方面, 作 品有不能完全脱离接收者的审美经验使期 待视野完全处 
于陌生的状态, 这就会造成创作曲高和寡, 使之成为阳春 白雪之作品。其二, 受众对于同一个作品有着不同的期待 视野, 使不同读者对于作品的好恶参半。这正是由于接受 者的文化修养、审美素养、生活阅历、生活经验影响下造 成的, 对同一作品大家会有不同审美感受, 同时赋予作品 的意义也不尽相同。

\section{4. 非遗文创产品中意象与创新}

中国古典美学范畴中的意境与荣格集体无意识、原始 意象有着异曲同工之 妙, 两者都通过象征、隐喻的方式 来进行信息的交流与传达，从而扩大接受者的 审美想象 空间, 产生无尽的联想。在当代非遗文创产品中, 设计师 利用传统神话 符号、象征文字、图案装饰来传递文化信 息, 这不仅彰显了中国悠久的传统文化, 而且通过文创 这一载体激发了人们集体无意识使之外显出来。

\section{1. 非遗文创中的意象}

中国绚丽多姿的艺术文化宝库中无一不追求意境,一 种虚实相生的审美意境, 并且昭示某种传统意象, 这种昭 示的意象同荣格的原型有这异曲同工之妙，这些传统意 象通过物质媒介和装饰图案等感性材料得以显现, , 即荣 格的原始意象是相通的。中国的传统意象通过书法与绘画 等最有代表特色的媒介得以一直继承下去, 书法是中国特 有的艺术文化种类, 首先文字本身就具有象征意义, 能够 传递信息和交流, 其次书法的结构、章法、风格更是能彰 显一种人物的一种品格与精神的含义。宗白华先生曾在 《世说新语》中说到: “晋人风神萧洒, 不滞与物, 这优 美的自由的心灵找到一种最适宜于表现他自己的艺术, 这 就是书法中的行草。 ${ }^{[5]}$ ” 中国绘画以其独立的绘画体系相 别与西方绘画, 西方绘画以焦点透视为主讲究写实, 而中 国绘画讲究散点透视, 并且画中常有留白, 给予人无尽的 遐想空间, 宋代绘画中的 “马一角, 夏半边” 不仅体现了 宋画的意境，而且丰富了审 美想象空间。

在当下文创产品中，绘画与书法是以不同的面貌出 现，而且将中华民族的集 体无意识在当下传承下去，在 中国台北故宫博物院中最畅销的 “朕知道了胶带”，简简 单单的四个字却激发了人们的强烈兴趣。这正是康熙批注 奏折真迹的复, 由于康熙在批阅奏折时, 经常在奏折末用 批注 “朕知道了” 所以台北故宫以书法为媒介, 胶带为其 载体, 设计了该文创产品, 而且产品采用红、白、黄三色, 其配色分别引发人们脑海中构建出在朱墙、书法、皇室等 意象, 并且引发了人们回顾中华悠久的历史文化; 北京故 宫博物院是中国最大的古代历史、文化、艺术博物馆, 并 且同时是世界文化遗产, 不仅相继推出了文创产品, 而且 倡导一种具有中国特色生活美学，通过文创传承中华文 化, 其通过王希孟的绘画《千里江山图》推出了一系列的 文创产品, 图案不仅是中国传统绘画的体现, 而且引发了 人们无意识中对意境的追求, 使之成为显性。总之, 正如 原型需要一定的感性材料和原始意象来引发一样, 中国的
传统意象正是通过一定的媒介得以在信息时代继承下来。

\section{2. 非遗文创中的创新}

人们的生活方式相比之前发生了翻天覆地的变化, 原 始的感性材料已经不能充分满足人们的审美需要, 只有通 过创新非遗文化载体，才能使民族文化传承下 去，这同 时也是设计贵在创新的重要原因。在创新过程中, 我们要 结合当下的科 技成果把握创新程度与受众接受距离的问 题, 同时也要满足不同群体的审美需 求, 因为受众的审 美经验以一种集体无意识的形式存在于心理之中, 古代的 神话 在不同的时代也采用了不同的艺术载体，如舞蹈、 绘画、音乐、戏曲等中国传统文化, 使得传统意象弥久历 新。在信息技术革命后出现了新的艺术种类与形式, 如电 影、动画、流行音乐等。

舞蹈以肢体语言的方式进行情绪的表达、传达着传统 意象, 如孔雀在中国被当做是百鸟之冠, 有着吉祥如意的 寓意，傣族的孔雀舞就是模仿孔雀优雅的动作来进行表 演，同时传递和继承者中国的传统意象，中国著名民族舞 蹈艺术家杨丽 萍的孔雀舞，通过肢体语言并配合音乐表 演出孔雀的姿态，不仅体现了中国的文 化，而且潜移默 化的影响着人们的心理中的集体无意识; 在中国电影史 上，中国台湾著名导演李安的《卧虎藏龙》，其中由周润 发扮演的李慕白与章子怡扮演的玉娇龙的斗剑场景选在 竹林, 竹子一直以来都是中国传统诗画中重要的题材, 具 备有气节、刚直不阿的象征寓意，很好的祄托了李慕白 的大侠风范，经由竹子这一感性材料 可以找到其象征意 义与原型, 并且通过其产生联想, 使得竹子这一个感性材 料可 以通过新的艺术载体的体现，同时电影中的武术作 为中华非遗文化的传承是我 们民族世世代代积淀而成、 安魂守魄的武器, 中华武术伴随中国历史发展历经了上千 年的历史，成为维系中华民族生存与发展的纽带、和承载 中华儿女基因的重要因素, 中华武术俨然已经成为集体无 意识中的重要成分。在当代弘扬中国传统武术文化不仅能 够强生健体, 而且能够激起我们的文化自信, 是我们中国 优秀文化的重要组成部分。

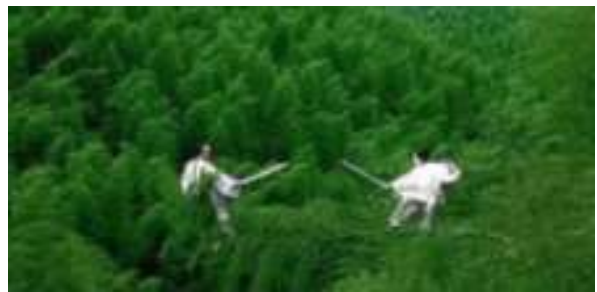

图 2 《卧虎藏龙》剧照

在非遗文创产品中，设计师采用具有象征意义的颜 色、符号、图案纹样等, 对产品进行设计, 唤醒人们的在 潜移默化的过程中形成的集体无意识, 同时这些 重要的 象征符号都是具有一定的文化含义又反过来影响人们现 有的审美经验, 新 的非遗文创产品形式的出现不断开拓 人们的审美空间，同时创新传统意象的媒介。 


\section{5. 非遗文创的中文化心理的传承}

在非遗文创产品中，图案、技艺、风俗习惯都是都是 中华文化中不同的原始意象或者说集体无意识以不同的 载体得以显现, 并且以新的形式继承下去。当代 非遗文 创产品不仅以传统的技艺、生活器具、图案纹样等作为载 体进入我们的生 活, 而且利用现代科技和新的艺术形式 进入我们的生活领域, 如电影艺术、动画、环境设计、 视觉传达等, 通过一系列的现代创新感性材料, 也就是荣 格说的原始 意象来引发我们心理中的集体无意识中的原 型, 但是与此同时要符合人民的期待 视野并且激起人们 心中对中华文化的波澜。

\section{1. 非遗文创的设计分析}

在非遗等产品设计的过程中, 我们要提取其原有原型 的象征意义, 并且将象征的符号用另外一种 “所指” 进行 传达, 首先在颜色方面, 中国自古以来都十分 讲究颜色 的搭配, 在封建社会中地位的不同, 对服饰、建筑、器具 等采用的色彩 也有了严格的区别, 各种颜色都有着其象 征寓意, 如黄色系代表帝王权贵、红色 系代表吉祥与正 统、青色系代表着蓬勃的生机、紫色代表的是祥瑞、庄重 之色等。虽然中国整体对颜色搭配的审美是有共识的, 但是因为中国少数民族众多且采用 “大杂居小聚居” 的 居住状况, 因此在不同的区域不同的色彩搭配也是十分讲 究 的, 如蒙古人就比较讨厌黑色。其次在图案方面, 每 种动物、植物也有着不同的 寓意如龙在中国传统文化中 是权势、高贵、尊荣的象征、锦鲤自古以来都有吉祥顺利 的的寓意。这些重要的原始意象都是集体无意识在各种设 计中的具体体现, 但是在当下时代我们需要不断创新影响 人们生活 的感性材料, 最后由设计师匠心独运的思维将 其整合, 并且诱发人们的期待视野, 最终增强中华民族 的文化自信。

在中国的对审美的集体意识中, 中国传统的审美思想 中李白倡导着一种 “清 水出芙蓉, 天然去雕饰”, 这样的 一种审美思想一直潜移默化的影响着人们对器 物、纹样 的设计, 纵观中华艺术设计宝库, 多数呈现着一种 “始发 芙蓉” 自然之美, 同时也提倡着 “天人合一” 的一种审美 观。在当下对非遗文创中消费者的调查过程中, 也可以 发现多数人可能选择的是少装饰的器物, 因此在中华五千 年文化积淀下的集体 无意识不仅影响着设计师的设计过 程，同时也作用于消费者的期待视野与集体无 意识。

\section{2. 英都灯笼的非遗文创调研}

福建南安英都的拔拔灯在正月初九举行, 传说这天是 玉帝的诞辰, 闽南地区俗称 “天公生”, 来自不同乡镇的 人群由贯穿着长绳的灯笼连接着, 并且聚集在昭惠庙前 面, 大家缓慢前行, 共同建构了一个灯的海洋, 在灯笼上 有着 “招财进宝” “兴旺” 等吉祥寓意的文字, 近百年来, 拔拔灯不仅成为了一项喜迎新春的重要民俗活动, 而且潜
移默化成为了人么你心理活动中的集体无意识, 但是与此 同时人们的对传统的文化也是抱有创新的态度, 因此姚斯 的期待视野对传统民俗的活动中灯笼的衍生产品设计是 必要的, 不仅激发人们对这种非遗传统活动认识, 而且设 计这项活动将这种传统活动带向更加广阔的文化天地, 让 身处在不同地区的人增强对中国传统非遗的认同感。

\section{3. 英都非遗文创设计实践}

伴随着现在人们生活方式的迅速更迭, 出现了许多新 形式的生活用具, 编者通过调查福建南安英都每年正月初 九的拔拔灯民俗活动, 针对拔拔灯活动中灯笼的原型与当 地人们对其的反应将灯笼的原型与餐具进行了结合设计, 围绕灯笼原型的衍生设计也开始逐渐开始多样化, 该设计 将灯笼的传统意象与整套餐具进行了结合, 在颜色上, 采 用象征吉祥、喜庆灯笼的红色, 将组合设计与模块化设计 的思路运用到现代人的餐具上, 通过创新传统餐具设计来 迎合人们心中的期待视野, 并且通过整体造型来激发人们 集体无意识, 使集体无意识在潜移默化之下得到继承与发 展。

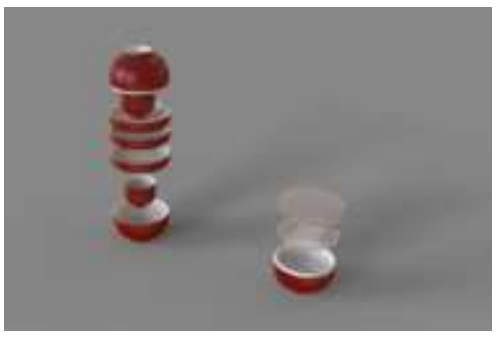

图 3 作者设计实践

\section{6. 结语}

当代非遗文创产品不仅体现中国文化的传统意象, 而 且新的文创产品形式的 出现开始扩大人们的审美想象空 间, 创新人们的审美经验。在各种大众文化进入 中国后, 非遗文创不仅继承和发展了中华文化中的集体无意识, 对 人们的期待视 野重新定义, 而且开发经济增长的新模式, 正如北京故宫博物院线上与线下文创产品全年总收入由 2013 年的六亿元增长到 2017 年的十五亿元。 ${ }^{[6]}$ 传统的 技艺、文化、风俗在非遗文创产品的设计制造过程中重新 焕发生机, 带动文创产品等手工业发展, 提供了更多的就 业机会。

当前整个社会都在倡导非遗文化的弘扬, 对无形的传 统文化与工艺的发掘与 开发时不可待, 这在一定程度上 有助于中国文化产业的发展和文化的输出。通过 现存风 俗习惯、技艺等非物质文化的调查与研究并且结合当代最 新科技成果应用 成果, 使古老的中国传统意象潜移默化 的流传下去。在未来文化产业如何更好设 计和制作非遗 文创产品, 尚需各行业的人员相互合作、交流，如设计师、 社会学 家、历史学家、经济学家、企业家等, 这样才能 不曲解文化、风俗习惯陷入桎梏 里面, 并且继承和发展 
优秀的传统文化, 坚定文化自信的道路。

\section{REFERENCES}

[1] Carl Gustav Jung. (1987) On the relationship between analytical psychology and poetry. In: Feng, C. Su, K. (Eds.), Psychology and Literature. SDX Joint Publishing Company. Beijing. pp.113.

[2] Carl Gustav Jung. (2011) Analytical psychology its theory and practice. Yilin press publishing. Jiangsu.

[3] Hu J.Z, Wang YC, Li Y.Z.(2016)Chapter 4 Jung analysis psychology theory and its literary thought.Hu J.Z, Wang Y.Z, Li Y.Z.(Eds.),The Classic Writing of Western Literature Thoery. Peking University Press.Beijing.pp100.

[4] Hans Robert Jauss. (1987) Receptional Aesthetic and Aesthetic Theory. Liaoning people's Publishing house. Shenyang.

[5] Zong Baihua. (1970) A New Account of Talls of the World. ZHONGHUA Book Company. Beijing.

[6] Sina Finance CN. (2019) The Forbidden City has exposed itself! Its cultural and creative products revenue reached 1.5 billion yuan in 2017. http://finance.sina.com.cn/roll/2019-02-19/doc-ihqfskcp65 68160.shtml. 